\title{
Obesogenic Behavior and Binge Eating Disorder in an Elderly Female with Schizophrenia
}

\author{
Vishnupriya Veeraraghavan* \\ Department of Psychiatry, Aarupadai Veedu Medical College and Hospital, Puducherry, India
}

Eating disorders, like binge eating, have a strong association with schizophrenia. Illness characteristics like disordered eating, cognition, and behavior can lead to eating disorders. Previous research highlighted the neurobiological structural similarity and the role of hormonal factors, like hypocretin, in the etiology of eating disorders in schizophrenia. Modifying the obesogenic environment by adapting healthy eating styles has been effective in reducing binging episodes. Antipsychotic medications also have a role in altering eating patterns that result in binge eating disorder. Adolescents with psychosis have a higher incidence of eating disorders. Here, we present an elderly female with schizophrenia who had obesogenic behaviors along with binge eating disorder. Interestingly, the patient had atypical age of onset and presentation and no psychopathological symptoms as a reason for binging.

Key words: Obesity, Schizophrenia, Binge-eating disorder, Serotonin reuptake inhibitors

\author{
Received September 22, 2020 \\ Reviewed October 31, 2020 \\ Accepted March 18, 2021 \\ ${ }^{*}$ Corresponding author \\ Vishnupriya Veeraraghavan \\ (iD) \\ https://orcid.org/0000-0001-6279-5556 \\ Department of Psychiatry, Aarupadai \\ Veedu Medical College and Hospital, \\ No. 22, Moolakulam, Puducherry \\ 605010, India \\ Tel: $+91-9499942564$ \\ Fax: +91-9499942564 \\ E-mail:vishnu27raghav@gmail.com
}

\section{INTRODUCTION}

Obesogenic behaviors refer to a spectrum of eating behaviors that include "inability to leave food behind," "anger when people comment on their eating," "craving for food," and "night eating." Binge eating disorder is characterized by consumption of large amounts of food in a relatively short time period at least once a week for 3 months and is accompanied by a sensation of losing control over eating behavior. Furthermore, binge eating disorder is accompanied by eating more rapidly than normal, eating until uncomfortably full, eating large amounts of food when not feeling hungry, eating alone because of being embarrassed by how much one is eating, and feeling disgusted with oneself after overeating. ${ }^{2}$ An episode of binge eating is defined as "eating, in a discrete period of time, an amount of food that is definitely larger than most of the people would eat in a similar period of time under similar circumstances" according to the Diagnostic and Statistical Manual. ${ }^{2}$ The discrete period of time is usually considered 2 hours, and lack of control over eating behaviors is commonly observed in these patients. Moreover, binge eating disorder is not associated with regular use of inappropriate compensatory behaviors like purging. ${ }^{3}$

One study showed that $16 \%$ of people with schizophrenia had binge eating disorder, ${ }^{4}$ and in China, Japan, Africa, and Latin America, binge eating disorder had the highest prevalence among all eating disorders, although no cases were reported in India. ${ }^{5}$ In India, the most common psychiatric comorbidity (50\%) observed in patients with an eating disorder was depression. ${ }^{5}$ Here, we present an interesting case of schizophrenia in a patient whose psychotic features were under control, but her eating habits were worrisome and prompted consultation. 


\section{CASE REPORT}

A 63-year-old female was brought by her husband and son to the psychiatry outpatient department with the complaint of excessive eating for the previous 6 months. Her past medical history included type 2 diabetes mellitus for the past 10 years. Her diet consisted of the following South Indian food items along with curd: (1) dosas: 20-25 per day; 3 every 2 waking hours and once or twice during the nighttime; approximate calories, 2,000-2,500 per day; (2) chapathi: 20-25 per day; 3 every 2 waking hours and once or twice during the nighttime; approximate calories, 1,800-2,000 per day; and (3) cooked rice with sambar: 7-8 bowls per day; 1 every 3 hours and once during the nighttime; approximate calories, 1,5001,700 per day.

She had been diagnosed with schizophrenia 20 years previously and was on irregular treatment. For the past 2.5 years, she had used risperidone $4 \mathrm{mg}$ and amisulpride $50 \mathrm{mg}$ tablets by mouth to help control her abnormal behavior. Six months before presentation, her eating patterns changed, and she started to consume more food than usual. Furthermore, she began to eat at night, one or two episodes, which hampered her sleep. When her family members tried to restrict her food intake, she became excessively irritable and quarrelsome. She commented that she could not control her hunger and did not experience satiety, even after consuming large amounts of food. She did not attribute her symptoms to any thought or perceptual disturbance.

She was admitted to the psychiatric ward. On examination, she did not have any focal neurological signs or symptoms, and no repetitive behaviors, abnormal behaviors, or any other frontal release signs were observed. Her weight was $90 \mathrm{~kg}$. Her body mass index was $35 \mathrm{~kg} / \mathrm{m}^{2}$. Complete blood count, serum electrolytes, and tests for liver and renal function were all within normal limits. Her glycosylated hemoglobin level demonstrated good glycemic control. Neuroimaging magnetic resonance imaging (MRI) revealed cortical atrophic changes and lacunar infarcts. Thyroid function test and serum vitamin B12 level were normal. The following psychological scales were administered: (1) Mini Mental State Examination score was 27/30, (2) Brief Psychiatric Rating Scale score was 35, and (3) Binge Eating Scale score was 28.

A neurologist, whose opinion was sought in view of the atrophic changes and lacunar infarcts on MRI, advised tablet aspirin/atorvastatin 75/10 mg and considered the atrophic changes age-related and incidental as there were no symptoms of forgetfulness or any abnormal movements. A medical gastroenterologist advised serotonergic agents for mitigating her hunger. She was started on capsule fluoxetine $20 \mathrm{mg}$ once daily and tablet topiramate $25 \mathrm{mg}$ at night, and tablet risperidone was cross tapered with tablet aripiprazole $10 \mathrm{mg}$ at night. Recording and maintenance of a diet chart were required for supervision of the patient's diet. Dietary restrictions of reduced quantity of single meals and increased duration between meals (from 2 hours to 4 hours) were practiced. After 2 weeks of treatment, her meal frequency decreased to 4-5 times a day, and the quantity of food per meal was reduced. Her feelings of suspiciousness reduced, and she would only occasionally report it.

However, she was not able to manage her hunger at times and would demand food. So, the dosage of fluoxetine was increased to $40 \mathrm{mg}$ and topiramate was increased to $50 \mathrm{mg}$. After 10 days, her meal frequency decreased to 3-4 meals per day, and the nighttime eating stopped completely. Her feeling of hunger and demanding food also stopped. She experienced a weight loss of $2.5 \mathrm{~kg}$. She was discharged with the following medications: $40 \mathrm{mg}$ fluoxetine, $50 \mathrm{mg}$ topiramate, and $10 \mathrm{mg}$ aripiprazole. At a follow-up visit 3 weeks later, did not complain of excessive hunger or nighttime eating. She had lost another $1.5 \mathrm{~kg}$ of weight. The fluoxetine and topiramate were slowly tapered and stopped, and her family members were educated regarding the need for good glycemic control and physical activity. Informed consent was obtained from the patient. Ethical committee waiver off was obtained from the institution as it is a case report.

\section{DISCUSSION}

Obesogenic behavior in the elderly is associated with increased risk of cardiometabolic syndrome, dementia, and impaired quality of life. ${ }^{6}$ Previous study by Sharma ${ }^{7}$ revealed that physical activity in obese elderly individuals can prevent the onset of dementia. Despite a growing interest in weight gain and metabolic disorders in individuals with schizophrenia, evaluation of eating disorders and obesogenic behavior remains underexplored. ${ }^{8}$ Previous researchers have highlighted the role of olanzapine and clozapine in inducing 
eating disorders, ${ }^{9}$ whereas our patient developed a disordered eating habit while using risperidone. Antipsychotics antagonize histamine $\mathrm{H} 1$, serotonin $2 \mathrm{~A} / 2 \mathrm{C}$ (5-hydroxytryptamine [5-HT]2A/C), and dopamine D1/D2/D3 receptors as well as adrenergic and muscarinic receptors. Serotonergic and muscarinic receptor antagonism has been implied in disruption of insulin homeostasis and can lead to antipsychotic-induced weight gain. ${ }^{10}$ The serotonergic system is involved in antipsychotic-induced binge eating because both drugs affect serotonergic neurotransmission as an antagonist with high affinity for all 5-HT2 receptor subtypes and the 5-HT6 receptor. Altered 5-HT physiology, seen in patients treated with antipsychotics, predisposes patients to this abnormal eating behavior. ${ }^{11}$ Our patient was receiving an antipsychotic and is considered a case of antipsychotic-induced eating disorder. ${ }^{12}$ An obesogenic environment can be changed by "modifying the food delivery" and "adjusting the offered food,"11 which was successful in our patient. Previous studies in patients with eating disorders have shown structural changes, like cortical atrophy, especially in the inferior frontal regions. ${ }^{13}$ Our patient had cortical atrophy without any memory deficits. Previous studies suggest that 5-HT (serotonin) neurotransmission leads to reduced eating behavior, whereas reduced 5-HT activity precipitates compulsive or binge eating. Hence, serotonin reuptake inhibitors (SRIs) can be used to control binge eating by increasing serotonergic neurotransmission. ${ }^{14}$ Theories postulate that stimulation of the lateral hypothalamus by glutamate and glutamate agonists, including kainite or AMPA ( $\alpha$-amino-3-hydroxy5-methyl-4-isoxazolepropionic acid) agonists, causes a rapid increase in food intake. ${ }^{15}$ Topiramate is an antagonist at kainite and AMPA glutamate receptors and helps in reducing binging. ${ }^{16}$ Few randomized trials highlighted that topiramate produces a significant weight loss of 6-8 kg over a period of 24 weeks, with improvement in lipid parameters, glycemic control, and blood pressure. Topiramate also augments the effects of cognitive behavioral therapy in patients with binge eating. ${ }^{17}$ Topiramate can be used as monotherapy or in combination with phentermine for weight loss. ${ }^{18}$ Selective SRIs, like fluoxetine, were found to reduce binge episodes. ${ }^{19}$ Atypical antipsychotics, like aripiprazole, when combined with SRIs also were effective in reducing binge episodes. ${ }^{20}$ Finally, previous studies described underreporting of dietary calorie intake as a common feature in obese individuals, but this was not found in our patient.
This report described a case of binge eating disorder in a patient with schizophrenia who was on antipsychotics. In India, there is an alarming increase of obesity. For patients with a high degree of body dissatisfaction, psychoeducation in the form of diet therapy and moderate calorie restrictions must be encouraged by the treating physician, for both patients and their family members. Weight loss before binge stabilization and attempts to exclude certain food items should be discouraged. ${ }^{20}$ Based on our case report and previous studies in obese individuals, we suggest that asking about eating behavior is more reliable than asking about quantity of food consumed. This distinguishes eating disorders and food addiction from other substance addictions and dependence disorders.

\section{CONFLICTS OF INTEREST}

The author declares no conflict of interest.

\section{REFERENCES}

1. Aronoff J. Eat to live light [Internet]. Live Light; 1992 [cited 2021 Mar 18]. Available from: https://www.myjanee.com/ livelight/eat/plates.htm

2. American Psychiatric Association. Diagnostic and statistical manual of mental disorders (DSM-5). 5th ed. Arlington (VA): American Psychiatric Association; 2013.

3. Cooper Z, Fairburn CG. Refining the definition of binge eating disorder and nonpurging bulimia nervosa. Int $\mathrm{J}$ Eat Disord 2003;34 Suppl:S89-95.

4. Ramacciotti CE, Paoli RA, Catena M, Ciapparelli A, Dell'Osso L, Schulte F, et al. Schizophrenia and binge-eating disorders. J Clin Psychiatry 2004;65:1016-7.

5. Vaidyanathan S, Kuppili PP, Menon V. Eating disorders: an overview of indian research. Indian J Psychol Med 2019;41: 311-7.

6. Janssen I, Mark AE. Elevated body mass index and mortality risk in the elderly. Obes Rev 2007;8:41-59.

7. Sharma AM. Physicians' calling patients on excess weight may provide reality check and increase desire to lose weight in overweight and obese individuals. Evid Based Med 2011;16: 176-7. 
8. Kouidrat Y, Amad A, Lalau JD, Loas G. Eating disorders in schizophrenia: implications for research and management. Schizophr Res Treatment 2014;2014:791573.

9. Brömel T, Blum WF, Ziegler A, Schulz E, Bender M, Fleischhaker $\mathrm{C}$, et al. Serum leptin levels increase rapidly after initiation of clozapine therapy. Mol Psychiatry 1998;3:76-80.

10. Raben AT, Marshe VS, Chintoh A, Gorbovskaya I, Müller DJ, Hahn MK. The complex relationship between antipsychoticinduced weight gain and therapeutic benefits: a systematic review and implications for treatment. Front Neurosci 2018;11: 741.

11. Kluge M, Schuld A, Himmerich H, Dalal M, Schacht A, Wehmeier PM, et al. Clozapine and olanzapine are associated with food craving and binge eating: results from a randomized double-blind study. J Clin Psychopharmacol 2007;27:662-6.

12. Milano W, Capasso A. Psychopharmacological options in the multidisciplinary and multidimensional treatment of eating disorders. Open Neurol J 2019;13:22-31.

13. Looijmans A, Stiekema AP, Bruggeman R, van der Meer L, Stolk RP, Schoevers RA, et al. Changing the obesogenic environment to improve cardiometabolic health in residential patients with a severe mental illness: cluster randomised controlled trial. Br J Psychiatry 2017;211:296-303.

14. Donnelly B, Touyz S, Hay P, Burton A, Russell J, Caterson I. Neuroimaging in bulimia nervosa and binge eating disorder: a systematic review. J Eat Disord 2018;6:3.

15. Blundell JE. Serotonin manipulations and the structure of feeding behaviour. Appetite 1986;7 Suppl:39-56.

16. Stanley BG, Ha LH, Spears LC, Dee MG 2nd. Lateral hypothalamic injections of glutamate, kainic acid, D,L-alpha-amino3-hydroxy-5-methyl-isoxazole propionic acid or N-methyl-Daspartic acid rapidly elicit intense transient eating in rats. Brain Res 1993;613:88-95.

17. Bray GA, Hollander P, Klein S, Kushner R, Levy B, Fitchet M, et al. A 6-month randomized, placebo-controlled, dose-ranging trial of topiramate for weight loss in obesity. Obes Res 2003;11:722-33.

18. Kim BY, Kang SM, Kang JH, Kim KK, Kim B, Kim SJ, et al. Current long-term pharmacotherapies for the management of obesity. J Obes Metab Syndr 2020;29:99-109.

19. Claudino AM, de Oliveira IR, Appolinario JC, Cordás TA, Duchesne M, Sichieri R, et al. Double-blind, randomized, placebo-controlled trial of topiramate plus cognitive-behavior therapy in binge-eating disorder. J Clin Psychiatry 2007;68: 1324-32.

20. McElroy SL, Guerdjikova AI, Mori N, O’Melia AM. Pharmacological management of binge eating disorder: current and emerging treatment options. Ther Clin Risk Manag 2012;8: 219-41. 